\title{
Transferences of Purkinje systems
}

\section{WF Harris}

\author{
Department of Optometry, University of Johannesburg, PO Box 524, Auckland Park, 2006 South \\ Africa
}

<wharris@uj.ac.za>

Received 31 August 2010; revised version accepted 23 March 2011

\begin{abstract}
The transferences of heterocentric astigmatic Purkinje systems are special: submatrices $\mathbf{B}$ and $\mathbf{C}$, that is, the disjugacy and the divergence of the system, are symmetric and submatrix $\mathbf{D}$ (the divarication) is the transpose of submatrix A (the dilation). It is the primary purpose of this paper to provide a proof. The paper also derives other relationships
\end{abstract}

among the fundamental properties and compact expressions for the transference and optical axis locator of a Purkinje system. (S Afr Optom 2011 70(2) 57-60)

Key words: Purkinje system, transference, symplecticity, heterocentricity, astigmatism, optical axis

\section{Introduction}

A recent paper $^{1}$ shows how to calculate the ray transference of astigmatic heterocentric catadioptric systems if the optical elements within the system are known. Purkinje systems, that is, the four systems responsible for formation of the Purkinje images observed in an eye, were treated as examples. Inspection of the those transferences calculated for a particular eye (see Table 1) suggests a number of features that may be common to all Purkinje and Purkinje-like systems, namely that the disjugacy and divergence are both symmetric and that the divarication is the transpose of the dilation. The purpose of this paper is to prove that all such systems do, indeed, have those features, and a couple more, and to derive compact forms for the transference and optical axis locator of a Purkinje system.

\section{Purkinje systems}

In forming a Purkinje image light is reflected off the front of one of the surfaces in the eye. That surface is acting as an anterior catoptric system which we represent by $\mathrm{S}_{\mathrm{c}}$; it is one of three subsystems that we identify. The other two consist of the same physical system, namely the portion of the eye in front of the reflecting surface, but traversed twice, once in the positive sense of the longitudinal axis $Z$ (Figure 1), and once in the negative sense. The portion traversed in the positive sense we represent as (unreversed) dioptric subsystem $\mathrm{S}_{1}$, the same portion traversed in the reverse sense we represent as reversed dioptric subsystem $\mathrm{S}_{1}^{\leftarrow}$. The Purkinje system itself can be represented as the three subsystems arranged sequentially as follows: ${ }^{1}$

$$
\mathrm{S}_{\mathrm{P}}=\mathrm{S}_{1} \mathrm{~S}_{\mathrm{c}} \mathrm{S}_{1}^{\Leftarrow} \text {. }
$$

The reflecting surface is the first surface of the tear film in the case of the first Purkinje system, the second surface of the cornea in the case of the second Purkinje system, the first surface of the lens in the case of the third Purkinje system and the back of the lens in the case of the fourth Purkinje system. In the case of the first Purkinje system subsystem $S_{1}$ is taken 
to be the trivial or identity system. ${ }^{1}$

Table 1 Transferences of the four Purkinje systems for a particular model eye. The trivial bottom rows are omitted. The top-right block is in millimeters and the bottom-left block is in kilodioptres.

$\mathbf{T}_{\mathrm{P}_{1}}=\left(\begin{array}{cccccc}1 & 0 & 0 & 0 & 0 \\ 0 & 1 & 0 & 0 & 0 \\ 0.2632 & 0 & 1 & 0 & 0.0200 \\ 0 & 0.2500 & 0 & 1 & 0.0400\end{array}\right)$
$\mathbf{T}_{\mathrm{P}_{2}}=\left(\begin{array}{cccccc}1.1269 & 0.0033 & 0.8667 & 0.0013 & -0.0033 \\ 0.0033 & 1.1253 & 0.0013 & 0.8651 & -0.0285 \\ 0.3116 & 0.0080 & 1.1269 & 0.0033 & -0.0080 \\ 0.0080 & 0.3079 & 0.0033 & 1.1253 & -0.0701\end{array}\right)$
$\mathbf{T}_{\mathrm{P}_{3}}=\left(\begin{array}{cccccc}1.3864 & 0.0466 & 7.2674 & 0.1391 & -0.3159 \\ 0.0462 & 1.4034 & 0.1391 & 7.2756 & 0.0827 \\ 0.1267 & 0.0153 & 1.3864 & 0.0462 & -0.1036 \\ 0.0153 & 0.1333 & 0.0466 & 1.4034 & 0.0273\end{array}\right)$
$\mathbf{T}_{\mathrm{P}_{4}}=\left(\begin{array}{cccccc}-1.2271 & -0.1662 & -1.6771 & -1.2068 & -0.2775 \\ -0.1631 & -1.8057 & -1.2068 & -5.8481 & 0.3561 \\ -0.3016 & -0.0224 & -1.2271 & -0.1631 & -0.0376 \\ -0.0224 & -0.3866 & -0.1662 & -1.8057 & 0.0491\end{array}\right)$

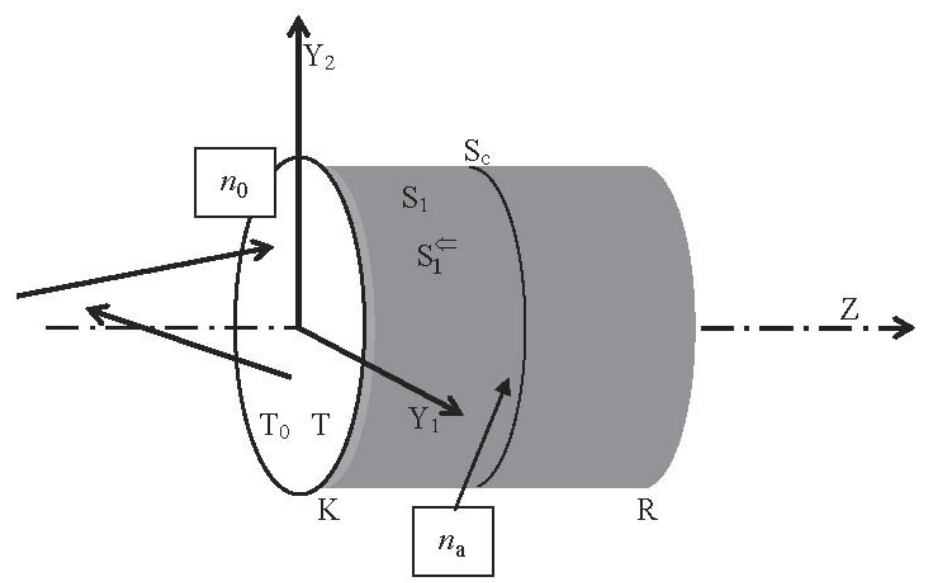

Figure 1 Schematic representation of Purkinje system $\mathrm{S}_{\mathrm{P}}=\mathrm{S}_{1} \mathrm{~S}_{\mathrm{c}} \mathrm{S}_{1}^{\Leftarrow}$. The eye is from the tear film on the cornea $\mathrm{K}$ to the retina $R$. $Z$ is a longitudinal axis. $Y_{1}$ and $Y_{2}$ are two mutually orthogonal transverse axes. $\mathrm{T}_{0}$ is a transverse plane immediately in front of the tear film; it represents the entrance plane of the Purkinje system. The exit plane $\mathrm{T}$ coincides with $\mathrm{T}_{0}$; hence the distance $z$ between them is 0 . The incident and emergent segments of a ray are shown. The ray traverses dioptric subsystem $\mathrm{S}_{1}^{\Leftarrow}$, is reflected by anterior catoptric subsystem and finally traverses reversed dioptric subsystem. $n_{0}$ and $n_{\mathrm{a}}$ are the indices immediately to the left of $\mathrm{T}_{0}$ and $\mathrm{S}_{\mathrm{c}}$ respectively.

\section{Transferences}

The general form of the transference of a catadioptric system is ${ }^{1}$

$\mathbf{T}=\left(\begin{array}{ccc}\mathbf{A} & \mathbf{B} & \mathbf{e} \\ \mathbf{C} & \mathbf{D} & \boldsymbol{\pi} \\ \mathbf{o}^{\mathrm{T}} & \mathbf{o}^{\mathrm{T}} & 1\end{array}\right)$.

$\mathbf{T}$ is $5 \times 5, \mathbf{A}$, the dilation, $\mathbf{B}$, the disjugacy, $\mathbf{C}$, the divergence, and $\mathbf{D}$, the divarication, are $2 \times 2$, e, the transverse translation, and $\pi$, the deflectance, are $2 \times 1, \mathbf{o}$ is the $2 \times 1$ null matrix, and $\mathbf{o}^{\mathrm{T}}$ is its matrix transpose. $\mathbf{T}$ is an augmented symplectic matrix; that implies relationships among its submatrices, a summary of which is presented elsewhere. ${ }^{2}$ In particular we can represent the transference of a Purkinje system as

$\mathbf{T}_{\mathrm{P}}=\left(\begin{array}{ccc}\mathbf{A}_{\mathrm{P}} & \mathbf{B}_{\mathrm{P}} & \mathbf{e}_{\mathrm{P}} \\ \mathbf{C}_{\mathrm{P}} & \mathbf{D}_{\mathrm{P}} & \boldsymbol{\pi}_{\mathrm{P}} \\ \mathbf{o}^{\mathrm{T}} & \mathbf{o}^{\mathrm{T}} & 1\end{array}\right)$.

The bottom rows of the transferences in Table 1 are omitted to save space. Units are also omitted for the same reason; $\mathbf{B}_{\mathrm{p}}$ and $\mathbf{e}_{\mathrm{p}}$ are in millimeters and $\mathbf{C}_{\mathrm{p}}$ is in kilodioptres.

In terms of the transferences of the three subsystems the transference of the Purkinje system is given by 1

$\mathbf{T}_{\mathrm{P}}=\mathbf{T}_{1}^{\Leftarrow} \mathbf{T}_{\mathrm{c}} \mathbf{T}_{1}$.

$\mathbf{T}_{1}$ is obtained by adding subscripts 1 to Equation 2. $\mathbf{T}_{\mathrm{c}}$, an anterior catoptric transference, is given by Equation 53 of the previous paper ${ }^{1}$ :

$\mathbf{T}_{\mathrm{c}}=\left(\begin{array}{ccc}\mathbf{I} & \mathbf{O} & \mathbf{0} \\ 2 n_{\mathrm{a}} \mathbf{K} & \mathbf{I} & 2 n_{\mathrm{a}} \mathbf{m} \\ \mathbf{o}^{\mathrm{T}} & \mathbf{o}^{\mathrm{T}} & 1\end{array}\right)$

where $n_{\mathrm{a}}$ is the index of refraction of the medium immediately anterior to the reflecting surface and $\mathbf{K}$ and $\mathbf{m}$ are the curvature and tilt respectively of the reflecting surface. $\mathbf{K}$ is a symmetric $2 \times 2$ matrix and $\mathbf{m}$ a $2 \times 1$ matrix. $\mathbf{T}_{1}^{\Leftarrow}$ is given by applying Equation 28 of the previous paper ${ }^{1}$ to subsystem $S_{1}$ :

$$
\mathbf{T}_{1}^{\Leftarrow}=\left(\begin{array}{ccc}
\mathbf{D}_{1}^{\mathrm{T}} & \mathbf{B}_{1}^{\mathrm{T}} & -\mathbf{D}_{1}^{\mathrm{T}} \mathbf{e}_{1}+\mathbf{B}_{1}^{\mathrm{T}} \boldsymbol{\pi}_{1} \\
\mathbf{C}_{1}^{\mathrm{T}} & \mathbf{A}_{1}^{\mathrm{T}} & -\mathbf{C}_{1}^{\mathrm{T}} \mathbf{e}_{1}+\mathbf{A}_{1}^{\mathrm{T}} \boldsymbol{\pi}_{1} \\
\mathbf{o}^{\mathrm{T}} & \mathbf{o}^{\mathrm{T}} & 1
\end{array}\right) .
$$


Substituting into Equation 4 one obtains

$$
\mathbf{T}_{\mathrm{P}}=\left(\begin{array}{ccc}
\mathbf{D}_{1}^{\mathrm{T}} \mathbf{A}_{1}+\mathbf{B}_{1}^{\mathrm{T}} \mathbf{C}_{1}+2 n_{\mathrm{a}} \mathbf{B}_{1}^{\mathrm{T}} \mathbf{K} \mathbf{A}_{1} & \mathbf{D}_{1}^{\mathrm{T}} \mathbf{B}_{1}+\mathbf{B}_{1}^{\mathrm{T}} \mathbf{D}_{1}+2 n_{\mathrm{a}} \mathbf{B}_{1}^{\mathrm{T}} \mathbf{K} \mathbf{B}_{1} & 2 \mathbf{B}_{1}^{\mathrm{T}}\left(n_{\mathrm{a}}\left(\mathbf{K} \mathbf{e}_{1}+\mathbf{m}\right)+\boldsymbol{\pi}_{1}\right) \\
\mathbf{C}_{1}^{\mathrm{T}} \mathbf{A}_{1}+\mathbf{A}_{1}^{\mathrm{T}} \mathbf{C}_{1}+2 n_{\mathrm{a}} \mathbf{A}_{1}^{\mathrm{T}} \mathbf{K} \mathbf{A}_{1} & \mathbf{C}_{1}^{\mathrm{T}} \mathbf{B}_{1}+\mathbf{A}_{1}^{\mathrm{T}} \mathbf{D}_{1}+2 n_{\mathrm{a}} \mathbf{A}_{1}^{\mathrm{T}} \mathbf{K} \mathbf{B}_{1} & 2 \mathbf{A}_{1}^{\mathrm{T}}\left(n_{\mathrm{a}}\left(\mathbf{K} \mathbf{e}_{1}+\mathbf{m}\right)+\pi_{1}\right) \\
\mathbf{o}^{\mathrm{T}} & \mathbf{o}^{\mathrm{T}} & 1
\end{array}\right) .
$$

From Equations 3 and 7 we see that a Purkinje system has disjugacy

$$
\mathbf{B}_{\mathrm{P}}=\mathbf{D}_{1}^{\mathrm{T}} \mathbf{B}_{1}+\mathbf{B}_{1}^{\mathrm{T}} \mathbf{D}_{1}+2 n_{\mathrm{a}} \mathbf{B}_{1}^{\mathrm{T}} \mathbf{K} \mathbf{B}_{1} \text {. }
$$

Now, because of symplecticity ${ }^{2}, \mathbf{D}_{1}^{\mathrm{T}} \mathbf{B}_{1}$ is symmetric. Because $\mathbf{K}$ is symmetric $\mathbf{B}_{1}^{\mathrm{T}} \mathbf{K} \mathbf{B}_{1}$ is symmetric as transposition shows. It follows that $\mathbf{B}_{\mathrm{p}}$ is symmetric and can be written

$$
\mathbf{B}_{\mathrm{P}}=2 \mathbf{B}_{1}^{\mathrm{T}}\left(\mathbf{D}_{1}+n_{\mathrm{a}} \mathbf{K} \mathbf{B}_{1}\right) \text {. }
$$

By a similar argument one finds that the divergence $\mathbf{C}_{\mathrm{p}}$ is also symmetric and can be written

$\mathbf{C}_{\mathrm{P}}=2 \mathbf{A}_{1}^{\mathrm{T}}\left(\mathbf{C}_{1}+n_{\mathrm{a}} \mathbf{K} \mathbf{A}_{1}\right)$.

The dilation of the Purkinje system is

$$
\mathbf{A}_{\mathrm{P}}=\mathbf{D}_{1}^{\mathrm{T}} \mathbf{A}_{1}+\mathbf{B}_{1}^{\mathrm{T}} \mathbf{C}_{1}+2 n_{\mathrm{a}} \mathbf{B}_{1}^{\mathrm{T}} \mathbf{K} \mathbf{A}_{1} \text {. }
$$

But because of symplecticity ${ }^{2}$

$$
\mathbf{D}_{1}^{\mathrm{T}} \mathbf{A}_{1}=\mathbf{B}_{1}^{\mathrm{T}} \mathbf{C}_{1}+\mathbf{I} \text {. }
$$

Hence

$$
\mathbf{A}_{\mathrm{P}}=2 \mathbf{B}_{1}^{\mathrm{T}} \mathbf{C}_{1}+2 n_{\mathrm{a}} \mathbf{B}_{1}^{\mathrm{T}} \mathbf{K} \mathbf{A}_{1}+\mathbf{I}
$$

which can be written

$$
\mathbf{A}_{\mathrm{P}}=2 \mathbf{B}_{1}^{\mathrm{T}}\left(\mathbf{C}_{1}+n_{\mathrm{a}} \mathbf{K} \mathbf{A}_{1}\right)+\mathbf{I} \text {. }
$$

Substitution from Equations 9, 10 and 14 and application of symplecticity shows that

$$
\mathbf{A}_{\mathrm{P}}^{2}=\mathbf{I}+\mathbf{B}_{\mathrm{P}} \mathbf{C}_{\mathrm{P}}
$$

from which it follows that

$$
\operatorname{det}\left(\mathbf{I}+\mathbf{B}_{\mathrm{P}} \mathbf{C}_{\mathrm{P}}\right) \geq 0 \text {. }
$$

Equation 15 shows that $\mathbf{A}_{\mathrm{p}}$ is a square root of $\mathbf{I}+\mathbf{B}_{\mathrm{P}} \mathbf{C}_{\mathrm{P}}$ and not necessarily the principal square root.

The divarication is

$$
\mathbf{D}_{\mathrm{P}}=\mathbf{C}_{1}^{\mathrm{T}} \mathbf{B}_{1}+\mathbf{A}_{1}^{\mathrm{T}} \mathbf{D}_{1}+2 n_{\mathrm{a}} \mathbf{A}_{1}^{\mathrm{T}} \mathbf{K} \mathbf{B}_{1} \text {. }
$$

Transposing Equation 12 and substituting we obtain

$$
\mathbf{D}_{\mathrm{P}}=2\left(\mathbf{C}_{1}^{\mathrm{T}}+n_{\mathrm{a}} \mathbf{A}_{1}^{\mathrm{T}} \mathbf{K}\right) \mathbf{B}_{1}+\mathbf{I} \text {. }
$$

Again using the fact that the curvature $\mathbf{K}$ is symmetric one finds from Equations 14 and 18 that
$\mathbf{D}_{\mathrm{P}}=\mathbf{A}_{\mathrm{P}}^{\mathrm{T}}$.

Applying the transposition of Equation 12 one finds that the divarication can also be written

$\mathbf{D}_{\mathrm{P}}=2 \mathbf{A}_{1}^{\mathrm{T}}\left(\mathbf{D}_{1}+n_{\mathrm{a}} \mathbf{K} \mathbf{B}_{1}\right)-\mathbf{I}$.

\section{Compact form of the transference}

Making use of Equations 9, 10, 14 and 20 one finds that the transference of a Purkinje system can be written more compactly as

$\left.\mathbf{T}_{\mathrm{P}}=2\left(\begin{array}{c}\mathbf{B}_{1}^{\mathrm{T}} \\ \mathbf{A}_{1}^{\mathrm{T}} \\ \mathbf{o}^{\mathrm{T}}\end{array}\right) \begin{array}{c}\left(\mathbf{C}_{1}+n_{\mathrm{a}} \mathbf{K} \mathbf{A}_{1}\right)^{\mathrm{T}} \\ \left(\mathbf{D}_{1}+n_{\mathrm{a}} \mathbf{K} \mathbf{B}_{1}\right)^{\mathrm{T}} \\ \left(n_{\mathrm{a}}\left(\mathbf{K} \mathbf{e}_{1}+\mathbf{m}\right)+\pi_{1}\right)^{\mathrm{T}}\end{array}\right)^{\mathrm{T}}+\mathbf{J}$

where

$\mathbf{J}=\left(\begin{array}{ccc}\mathbf{I} & \mathbf{O} & \mathbf{0} \\ \mathbf{O} & -\mathbf{I} & \mathbf{0} \\ \mathbf{0}^{\mathrm{T}} & \mathbf{0}^{\mathrm{T}} & 1\end{array}\right)$

is a matrix used before ${ }^{1}$.

It is also true for a Purkinje system that

$\mathbf{C}_{\mathrm{P}} \mathbf{e}_{\mathrm{P}}=\left(\mathbf{D}_{\mathrm{P}}-\mathbf{I}\right) \boldsymbol{\pi}_{\mathrm{P}}$,

a result one can show by substituting for $\mathbf{C}_{\mathrm{P}}, \mathbf{D}_{\mathrm{P}}, \mathbf{e}_{\mathrm{P}}$ and $\pi_{\mathrm{P}}$ from Equations 10, 18, 3 and 7 and recognizing the fact that both $\mathbf{A}_{1}^{\mathrm{T}} \mathbf{C}_{1}$ and $\mathbf{A}_{1} \mathbf{B}_{1}^{\mathrm{T}}$ are symmetric $^{2}$.

An expression has recently been derived for the optical axis locator $\mathbf{P}$ of a catadioptric system: Equation 17 of a previous pape ${ }^{3}$. Again substituting from Equations 9, 10, 14 and 20 the optical axis locator becomes

$$
\mathbf{P}=2\left(\begin{array}{ll}
\mathbf{B}_{1}^{\mathrm{T}}\left(\mathbf{C}_{1}+n_{\mathrm{a}} \mathbf{K} \mathbf{A}_{1}\right) & n_{0} \mathbf{B}_{1}^{\mathrm{T}}\left(\mathbf{D}_{1}+n_{\mathrm{a}} \mathbf{K ~ \mathbf { B } _ { 1 } )}\right. \\
\mathbf{A}_{1}^{\mathrm{T}}\left(\mathbf{C}_{1}+n_{\mathrm{a}} \mathbf{K} \mathbf{A}_{1}\right) & n_{0} \mathbf{A}_{1}^{\mathrm{T}}\left(\mathbf{D}_{1}+n_{\mathrm{a}} \mathbf{K} \mathbf{B}_{1}\right)
\end{array}\right)
$$

where $n_{0}$ is the index in front of the eye (usually 1 ). This can be written

$\mathbf{P}=2\left(\begin{array}{c}\mathbf{B}_{1}^{\mathrm{T}} \\ \mathbf{A}_{1}^{\mathrm{T}}\end{array}\right)\left(\begin{array}{c}\left(\mathbf{C}_{1}+n_{\mathrm{a}} \mathbf{K} \mathbf{A}_{1}\right)^{\mathrm{T}} \\ n_{0}\left(\mathbf{D}_{1}+n_{\mathrm{a}} \mathbf{K} \mathbf{B}_{1}\right)^{\mathrm{T}}\end{array}\right)^{\mathrm{T}}$. 
The right-hand side of Equation 25 is the product of a $4 \times 2$ and a $2 \times 4$ matrix. The rank of neither can exceed 2. But the rank of a product of matrices cannot be greater than the minimum of the ranks of the matrices being multiplied. ${ }^{4,5}$ It follows that $\operatorname{rankP} \leq 2$.

$\mathbf{P}$, then, is necessarily singular. (The ranks of the locators calculated before ${ }^{3}$ for the Purkinje systems of a particular model eye were 2.)

Inspection of the transferences listed in Table 1 suggests a gradually-changing character from the first to the third and a somewhat different character for the fourth Purkinje system. The entries in the first four columns are all positive or zero for the first three and all negative for the fourth. The dilation $\mathbf{A}_{\mathrm{p}}$ is an identity matrix in the case of the first Purkinje system and approximately scalar matrices in the case of the other Purkinje systems. The diagonal entries of $\mathbf{A}_{\mathrm{p}}$ increase from the first to the third Purkinje systems but those of the fourth Purkinje system are negative. For the first three Purkinje systems it turns out that $\mathbf{A}_{\mathrm{p}}$ is the principal square root of the right-hand side of Equation 15; for the fourth $\mathbf{A}_{\mathrm{p}}$ is the negative of the principal square root.

\section{Concluding remarks}

This paper has shown that, for a Purkinje system, the disjugacy $\mathbf{B}_{\mathrm{p}}$ and the divergence are symmetric and that the divarication $\mathbf{D}_{p}$ is the transpose of the dilation $\mathbf{A}_{\mathrm{p}}$. It has also derived other relationships among the fundamental properties of a Purkinje system (Equations 15, 16 and 23). The paper has provided a compact expression for the transference of a Purkinje system. An expression has been obtained for the optical axis locator of a Purkinje system; it shows that the locator is necessarily singular. This is a conclusion arrived at mathematically; the physical explanation depends on the fact that every ray arriving normal to the reflecting surface necessarily retraces the same path backward through the system and so defines one of an infinity of optical axes. Although the results obtained here are interesting in their own right some are likely to be useful computationally; for example, when estimating Purkinje transferences from multiple measurements made on an eye, one would want to build the particular features of the transferences into the algorithm.

\section{Acknowledgements}

I thank J R Cardoso, R D van Gool, T Evans, A Rubin and S D Mathebula for continuing discussions and the National Research Foundation for support.

\section{References}

1. Harris WF. Transferences of heterocentric astigmatic catadioptric systems including Purkinje systems. Optom Vis Sci 201087 778-786.

2. Harris WF. Symplecticity and relationships among the fundamental properties in linear optics. S Afr Optom 201069 3-13.

3. Harris WF. Optical axes of catadioptric systems including visual, Purkinje and other nonvisual systems of a heterocentric astigmatic eye. S Afr Optom 201069 152-160.

4. Lütkepohl H. Handbook of Matrices. Wiley, Chichester, 199661.

5. Bernstein DS. Matrix Mathematics. 2nd ed. Princeton Univ Press, Princeton, 2009104. 